definitions, then the loop integral $I(z, \beta)$ in (10) is developable asymptotically in the form

$$
I(z, \beta) \sim \sum_{n=0}^{\infty} \frac{c_{n}}{[\log (-[ \pm z])]^{\beta+n} \Gamma(1-\beta-n)} .
$$

It thus appears that the presence of an algebraic singularity of $g(w)$ presents no serious difficulty.

Purdue University

\title{
ON SOME FORMULAS INVOLVING THE DIVISOR FUNCTION
}

\section{HERBERT S. ZUCKERMAN}

Viggo Brun ${ }^{1}$ has proved the formulas

$$
\begin{aligned}
& \quad T_{1}(n)-T_{2}(n)+T_{3}(n)-\cdots=-\mu(n), \quad n>1, \\
& h(n)=T_{1}(n)-(1 / 2) T_{2}(n)+(1 / 3) T_{3}(n)-\cdots \\
& \quad=\left\{\begin{array}{l}
0 \text { if } n \text { is not a prime power, } \\
1 / t \text { if } n=p^{t}, p \text { a prime; }
\end{array}\right.
\end{aligned}
$$

where $T_{l}(n)$ is the number of ways that $n$ can be expressed as a product of $l$ factors, each greater than 1 . He obtains them as special cases of combinatorial theorems. Pavel $\mathrm{Kuhn}^{2}$ has also given proofs but it seems that no one has attempted to give elementary number theory proofs of these formulas. It is the purpose of this note to give such proofs and to point out a few other formulas similar to (1) and (2).

All the formulas which we shall prove can be proved very concisely by using the generating function

$$
\sum_{n=1}^{\infty} T_{l}(n) n^{-s}=\{\zeta(s)-1\}^{l},
$$

and some simple properties of the zeta-function. ${ }^{3}$ Our number theory

Received by the editors June 19, 1942.

${ }^{1}$ Netto, Lehrbuch der Combinatorik, 2d edition, 1927, chap. 14, especially pp. 276277.

${ }^{2}$ Det Kongelige Norske Videnskabers Selskab, Forhandlinger, 1939.

${ }^{3}$ Interchanging the order of summation we have $\sum_{n=1}^{\infty} \sum_{l=1}^{\infty}(-1)^{l-1} T_{l}(n) n^{-s}$ $=\sum_{l=1}^{\infty}(-1)^{l-1}\{\zeta(s)-1\}^{l}=-\zeta(s)^{-1}=-\sum_{n=1}^{\infty} \mu(n) n^{-s}$, and (1) is obtained by comparing coefficients of $n^{-8}$ in the two members. Similarly, (2) follows from $\sum_{n=1}^{\infty} \sum_{l=1}^{\infty}(-1)^{l-1} l^{-1} T_{l}(n) n^{-8}=\sum_{l=1}^{\infty}(-1)^{l-1} l^{-1}\{\zeta(s)-1\}^{l}=\log \zeta(s)=\sum_{p} \log \left(1-p^{-8}\right)^{-1}$ $=\sum_{p} \sum_{t=1}^{\infty} t^{-1} p^{-t s}$. 
proofs are the algebraic translations of these proofs.

In order to avoid certain special cases it will be convenient to define

$$
T_{0}(n)=\left\{\begin{array}{lll}
1 & \text { if } & n=1 \\
0 & \text { if } & n>1
\end{array}\right.
$$

The function $T_{1}(n)$ has already been defined as the number of ways $n$ can be expressed as a single factor greater than 1 , so we have

$$
T_{1}(n)=\left\{\begin{array}{lll}
0 & \text { if } & n=1 \\
1 & \text { if } & n>1
\end{array}\right.
$$

In writing an expression such as the left member of (1) it will be convenient to write it as $\sum_{l=1}^{\infty}(-1)^{l-1} T_{l}(n)$ but it should be noted that all the terms in the series are zero after a certain point. This will be true of all the series which we write in this way, so we are really dealing only with finite sums.

To prove (1) we first note that from the definition of $T_{l}(n)$ we have

$$
T_{l+1}(n)=\sum_{d \mid n, d \neq n} T_{l}(d)=\sum_{d \mid n} T_{l}(d)-T_{l}(n),
$$

for $l \geqq 0$. We let $f(n)=\sum_{l=0}^{\infty}(-1)^{l} T_{l}(n)$ and have, by (5),

$$
\sum_{d \mid n} f(d)=\sum_{l=0}^{\infty}(-1)^{l} \sum_{d \mid n} T_{l}(d)=\sum_{l=0}^{\infty}(-1)^{l}\left\{T_{l}(n)+T_{l+1}(n)\right\}=T_{0}(n) .
$$

Then by the Möbius inversion formula we have

$$
f(n)=\sum_{l=0}^{\infty}(-1)^{l} T_{l}(n)=\sum_{d \mid n} T_{0}(n / d) \mu(d)=\mu(n),
$$

where we have used (3). This is equivalent to (1).

This proof has an obvious extension. Putting $g(n)=\sum_{l=1}^{\infty}(-1)^{l}$ $\cdot(l+1) T_{l}(n)$ we have, using (5),

$$
\begin{aligned}
\sum_{d \mid n} g(d) & =\sum_{l=0}^{\infty}(-1)^{l}(l+1) \sum_{d \mid n}^{\infty} T_{l}(d) \\
& =\sum_{l=0}^{\infty}(-1)^{l}(l+1)\left\{T_{l}(n)+T_{l+1}(n)\right\} \\
& =\sum_{l=0}^{\infty}(-1)^{l} T_{l}(n)=f(n)=\mu(n) .
\end{aligned}
$$

Inverting this we obtain 


$$
g(n)=\sum_{l=0}^{\infty}(-1)^{l}(l+1) T_{l}(n)=\sum_{d \mid n} \mu(d) \mu(n / d)
$$

$$
=\left\{\begin{array}{l}
1 \text { if } n=1, \\
(-1)^{s} 2^{8} \text { if } n=p_{1} \cdots p_{s} q_{1}^{2} \cdots q_{t}^{2}, \\
0 \text { if } n \text { is divisible by a cube. }
\end{array}\right.
$$

In the same way we can prove

$$
\begin{aligned}
\sum_{l=0}^{\infty}(-1)^{l} & \frac{(l+1)(l+2)}{2} T_{l}(n)=\sum_{d \delta \mid n} \mu(d) \mu(\delta) \mu(n / d \delta) \\
= & \left\{\begin{array}{l}
1 \text { if } n=1, \\
(-1)^{s+u} 3^{s+t} \text { if } n=p_{1} \cdots p_{s} q_{1}^{2} \cdots q_{t}^{2} r_{1}^{3} \cdots r_{u}^{3}, \\
0 \text { if } n \text { is divisible by a fourth power. }
\end{array}\right.
\end{aligned}
$$

To prove (2) we need another expression for $T_{l}(n)$. From the definitions we have

$$
T_{l}(n)=\sum_{r_{1} \ldots r_{l}=n_{;} r_{i}>1} 1=\sum_{r_{1} \cdots \cdot r_{l}=n} T_{1}\left(r_{1}\right) \cdots T_{1}\left(r_{l}\right),
$$

for $l \geqq 1$. Multiplying this by $\log n$, rearranging, and using (9), we have

$$
\begin{aligned}
T_{l}(n) \log n & =\sum_{r_{1} \cdot r_{l}=n} T_{1}\left(r_{1}\right) \cdots T_{1}\left(r_{l}\right)\left(\log r_{1}+\cdots+\log r_{l}\right) \\
& =l \sum_{r_{1} \cdot \cdots r_{l}=n} T_{1}\left(r_{1}\right) \cdots T_{1}\left(r_{l}\right) \log r_{l} \\
& =l \sum_{r_{l} \mid n} T_{1}\left(r_{l}\right) \log r_{l} \sum_{r_{1} \cdots r_{l-1}=n / r_{l}} T_{1}\left(r_{1}\right) \cdots T_{1}\left(r_{l-1}\right) \\
& =l \sum_{r_{l} \mid n} T_{1}\left(r_{l}\right) \log r_{l} \cdot T_{l-1}\left(n / r_{l}\right) \\
& =l \sum_{d \mid n} T_{l-1}(d) T_{1}(n / d) \log (n / d) \\
& =l \log n \sum_{d \mid n} T_{l-1}(d) T_{1}(n / d)-l \sum_{d \mid n} T_{l-1}(d) T_{1}(n / d) \log d .
\end{aligned}
$$

Reducing this by (5) and (4) we have

$$
T_{l}(n) \log n=l T_{l}(n) \log n-l \sum_{d \mid n} T_{l-1}(d) T_{1}(n / d) \log d,
$$

which we can write in the form

$$
(1 /(l-1)) \sum_{d \mid n} T_{l-1}(d) T_{1}(n / d) \log d=(1 / l) T_{l}(n) \log n .
$$


If we now let

$$
h(n)=\sum_{l=1}^{\infty} \frac{(-1)^{l-1}}{l} T_{l}(n),
$$

we have, by (4) and (10),

$$
\begin{aligned}
\sum_{d \mid n} h(d) \log d & =\sum_{l=1}^{\infty}\left((-1)^{l-1} / l\right)\left\{\sum_{d \mid n} T_{l}(d) T_{1}(n / d) \log d+T_{l}(n) \log n\right\} \\
& =\sum_{l=1}^{\infty}(-1)^{l-1}\left\{(1 / l) T_{l}(n) \log n+(1 /(l+1)) T_{l+1}(n) \log n\right\} \\
& =T_{1}(n) \log n .
\end{aligned}
$$

Inverting this we obtain

$$
\begin{aligned}
h(n) \log n= & \sum_{d \mid n} T_{1}(n / d) \mu(d) \log n / d \\
= & \log n \sum_{d \mid n} T_{1}(n / d) \mu(d)-\sum_{d \mid n} T_{1}(n / d) \mu(d) \log d \\
= & \log n\left\{\sum_{d \mid n} \mu(d)-\mu(n)\right\} \\
& -\left\{\sum_{d \mid n} \mu(d) \log d-\mu(n) \log n\right\} \\
= & -\sum_{d \mid n} \mu(d) \log d,
\end{aligned}
$$

where we have used (4) and the fact that $\sum_{d \mid n} \mu(d)=0$ for $n>1$. Now it is easily verified that $-\sum_{d \mid n} \mu(d) \log d$ takes the value $\log p$ if $n=p^{t}$ and the value 0 if $n$ is not a prime power. ${ }^{4}$ Hence we have

$$
h(n)=\left\{\begin{array}{l}
1 / t \text { if } n=p^{t} \\
0 \text { if } n \text { is not a prime power, }
\end{array}\right.
$$

which is formula (2).

We can combine (6) and (12) to obtain another formula. We first note that, from (9), we have

$$
\begin{array}{rlrl}
T_{l}(n) & =\sum_{d \mid n}\left\{\sum_{r_{1}} \sum_{r_{\mu}=d} T_{1}\left(r_{1}\right) \cdots T_{1}\left(r_{\mu}\right) \sum_{r_{\mu+1}} \sum_{r_{l}=n / d} T_{1}\left(r_{\mu+1}\right) \cdots T_{1}\left(r_{l}\right)\right\} \\
& =\sum_{d \mid n} T_{\mu}(d) T_{l-\mu}(n / d), & 0 \leqq \mu \leqq l .
\end{array}
$$

\footnotetext{
${ }^{4}$ If $n=p^{r} m$ and $p$ does not divide $m$ we have $-\sum_{d \mid n} \mu(d) \log d=$ $-\sum_{\delta \mid m} \sum_{\gamma \mid p^{r}} \mu(\delta \gamma) \log (\delta \gamma)=-\sum_{\delta \mid m}\{\mu(\delta) \log \delta+\mu(\delta p) \log (\delta p)\}=-\sum_{\delta \mid m}\{\mu(\delta) \log \delta$ $-\mu(\delta) \log (\delta p)\}=\log p \sum_{\delta \mid m \mu}(\delta)$ and this has the value 0 if $m>1, \log p$ if $m=1$.
} 
We let

$$
\begin{aligned}
j(n)= & \sum_{l=1}^{\infty} \sum_{\mu=1}^{l}(-1)^{l-1}(1 / \mu) T_{l}(n) \\
= & T_{1}(n)-(3 / 2) T_{2}(n)+(11 / 6) T_{3}(n)-(25 / 12) T_{4}(n) \\
& +(137 / 60) T_{5}(n)-\cdots,
\end{aligned}
$$

and have, by (13),

$$
\begin{aligned}
j(n) & =\sum_{l=1}^{\infty} \sum_{\mu=1}^{l}(-1)^{l-1}(1 / \mu) \sum_{d \mid n} T_{\mu}(d) T_{l-\mu}(n / d) \\
& =\sum_{d \mid n} \sum_{l=1}^{\infty} \sum_{\mu+\nu=l ; \mu \geqq 1}(-1)^{\mu-1}(1 / \mu) T_{\mu}(d)(-1)^{\nu} T_{\nu}(n / d) \\
& =\sum_{d \mid n}\left\{\sum_{\mu=1}^{\infty}(-1)^{\mu-1}(1 / \mu) T_{\mu}(d) \sum_{\nu=0}^{\infty}(-1)^{\nu} T_{\nu}(n / d)\right\} \\
& =\sum_{d \mid n} h(d) f(n / d),
\end{aligned}
$$

where we have used (11) and (6). Then by (6) and (12) we have

$$
\begin{aligned}
j(n)= & \sum_{p^{t} \mid n}(1 / t) \mu\left(n / p^{t}\right) \\
= & \left\{\begin{array}{l}
0 \text { if } n \text { is divisible by the squares of two distinct primes, } \\
(-1)^{s+1} / t(t-1) \text { if } n=p^{t} q_{1} \cdots q_{s}, t>1, \\
(-1)^{s-1} s \text { if } n=q_{1} \cdots q_{s}
\end{array}\right.
\end{aligned}
$$

In quite a similar way we can prove

$$
\begin{aligned}
k(n)= & \sum_{l=2}^{\infty}(-1)^{l} \sum_{\nu+\mu=l ; \nu, \mu \geqq 1}(1 / \nu \mu) T_{l}(n) \\
= & T_{2}(n)-T_{3}(n)+(11 / 2) T_{4}(n)-(5 / 6) T_{\nu}(n) \\
& +(137 / 180) T_{6}(n)-\cdots \\
= & \left\{\begin{array}{l}
0 \text { if } n \text { has more than two distinct prime factors, } \\
2 / t u \text { if } n=p^{t} q^{u}, \\
\sum_{\nu=1}^{t-1}(1 / \nu(t-\nu)) \text { if } n=p^{t} .
\end{array}\right.
\end{aligned}
$$

Brun notes that $\sum_{n=1}^{m} T_{1}(n)=\{m\}, \quad \sum_{n=1}^{m} T_{2}(n)=\sum_{\mu=2}^{\infty}\{m / \mu\}$, $\sum_{n=1}^{m} T_{3}(n)=\sum_{\mu, \nu=2}^{\infty}\{m / \mu \nu\}$, and so on, where $\{x\}$ denotes the number of integers not less than 2 and not greater than $x$. Summing (2) he then has 


$$
\begin{aligned}
H(m)=\sum_{n=1}^{m} h(n)=\{m\} & -(1 / 2) \sum_{\mu=2}^{\infty}\{m / \mu\} \\
& +(1 / 3) \sum_{\mu, \nu=2}^{\infty}\{m / \mu \nu\}-\cdots .
\end{aligned}
$$

This formula can be used to determine $h(n)=H(n)-H(n-1)$ and hence, by (2), it gives a method (although impractical) of determining whether $n$ is a prime power. A similar thing can be done with each of our formulas. For example from (14) we have

$$
\begin{aligned}
J(m)=\sum_{n=1}^{m} j(n)=\{m\} & -(3 / 2) \sum_{\mu=2}^{\infty}\{m / \mu\} \\
& +(11 / 6) \sum_{\mu, \nu=2}^{\infty}\{m / \mu \nu\}-\cdots .
\end{aligned}
$$

Then $j(n)=J(n)-J(n-1)$, with the aid of (15), determines the number of prime factors of $n$ if $n$ is not divisible by the squares of more than one prime. As a numerical example consider $n=6$. We have

$$
\begin{aligned}
& J(6)=\{6\}-(3 / 2)[\{3\}+\{2\}]=1 / 2, \\
& J(5)=\{5\}-(3 / 2)\{5 / 2\}=5 / 2, \\
& j(6)=(1 / 2)-(5 / 2)=-2,
\end{aligned}
$$

and hence 6 has two distinct prime factors, each entering to the first power.

Brun also points out that from (2) we have

$$
H(m)=\sum_{n=1}^{m} h(n)=\sum_{t=1}^{\infty}(1 / t) \pi\left(m^{1 / t}\right) .
$$

The other formulas do not lead to such simple results but (16) gives

$$
K(m)=\sum_{n=1}^{m} k(n)=\sum_{\mu=1}^{\infty} \sum_{p^{\nu}}(1 / \mu \nu) \pi\left(\left(m / p^{\nu}\right)^{1 / \mu}\right) .
$$

If (10) is put into exponential form it can be stated in a more interesting way. From (10) we have

$$
\left[n^{T_{l}(n)}\right]^{1 / l}=\left[\prod_{d \mid n ; d \neq n} d^{T_{l-1}(d)}\right]^{1 /(l-1)} .
$$

The left member can be obtained by writing $n$ in all possible ways as a product of $l$ factors greater than 1, multiplying them together, 
and taking the $l$ th root. The right member can be obtained by writing all proper divisors of $n$ in all possible ways as products of $l-1$ factors greater than 1 , multiplying them all together, and taking the $(l-1)$ th root. Our result is that these two numbers are equal. Thus for $n=24$, $l=3$ we have

$[(2 \cdot 2 \cdot 6)(2 \cdot 6 \cdot 2)(6 \cdot 2 \cdot 2)(2 \cdot 3 \cdot 4)(2 \cdot 4 \cdot 3)(3 \cdot 2 \cdot 4)(3 \cdot 4 \cdot 2)$

$$
(4 \cdot 2 \cdot 3)(4 \cdot 3 \cdot 2)]^{1 / 3}=2^{9} \cdot 3^{3}
$$

$[(2 \cdot 2) \times(2 \cdot 3)(3 \cdot 2) \times(2 \cdot 4)(4 \cdot 2) \times(4 \cdot 3)(3 \cdot 4) \times(6 \cdot 2)(2 \cdot 6)]^{1 / 2}=2^{9} \cdot 3^{3}$.

\section{UNIVERSITY OF WASHINGTON}

